\title{
La lengua mayangna: a 30 años vista del comienzo de las actividades lingüísticas en la comunidad
}

\author{
Elena E. Benedicto \\ Elizabeth Salomón McClean
}

\section{SciELO Books / SciELO Livros / SciELO Libros}

BENEDICTO, E. E., and MCCLEAN, E. S. La lengua mayangna: a 30 años vista del comienzo de las actividades lingüísticas en la comunidad. In: HABOUD BUMACHAR, M., SÁNCHEZ AVENDAÑO, C., and GARCÉS VELÁSQUEZ, F., eds. Desplazamiento lingüístico y revitalización: reflexiones y metodologías emergentes [online]. Quito: Editorial AbyaYala, 2020, pp. 17-39. Desafíos en la Diversidad collection, n. 2. ISBN: 978-9978-10541-2. http://doi.org/10.7476/9789978105726.0002.

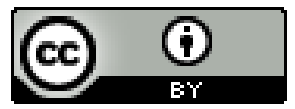

All the contents of this work, except where otherwise noted, is licensed under a Creative Commons Attribution 4.0 International license.

Todo o conteúdo deste trabalho, exceto quando houver ressalva, é publicado sob a licença Creative Commons Atribição 4.0.

Todo el contenido de esta obra, excepto donde se indique lo contrario, está bajo licencia de la licencia Creative Commons Reconocimento 4.0. 


\title{
La lengua mayangna: a 30 años vista del comienzo de las actividades lingüísticas en la comunidad ${ }^{1}$
}

\author{
Elena E. Benedicto² \\ Elizabeth Salomón McClean ${ }^{3}$
}

\begin{abstract}
Resumen
Nicaragua fue de los primeros países en Latinoamérica en implementar un programa de educación bilingüe para las poblaciones indígenas en su territorio, a pesar de problemas logísticos y económicos en el país. Ello sucedía a mediados de la década de 1980, a raíz de demandas de los pueblos indígenas a partir del triunfo de la Revolución en 1979.

La labor de descripción y documentación lingüística comienza ya desde ese momento, solicitada por el proceso de educación bilingüe que comenzaba. Desde entonces, el trabajo lingüístico se ha articulado con el desarrollo del programa PEBI, que fomenta el desarrollo de la lengua en la escuela, como lengua vehicular de contenidos y de cultura, y con otras instituciones educativas. En los años siguientes, el trabajo lingüístico se integró, siguiendo un enfoque participativo y de colaboración activa con la comunidad, en las universidades de nueva creación como URACCAN para desarrollar programas de formación lingüística a nivel universitario y proyectos de investigación científica.
\end{abstract}

Sin embargo, a pesar de contar con una coyuntura excepcional, los retos continúan, a veces en lugares insospechados. En este trabajo evaluamos cómo la incorporación del trabajo lingüístico dentro de un contexto estructural (instituciones, infraestructura, programas, etc...) dedicado al fomento de la lengua ha incidido en el mantenimiento, fortalecimiento y desarrollo de la lengua mayangna como elemento identitario y cultural de la comunidad mayangna.

1 Este trabajo está basado en la ponencia que se presentó en Salamanca 2018, con motivo del taller Desplazamiento Lingüistico y Revitalización: Reflexiones y Metodologías, parte del ICA 2018, bajo el título Las mil caras de un trabajo participativo durante 30 años: Nicaragua y los Mayangna. Los autores de esa presentación fueron Elena E. Benedicto (IELLab - Purdue University) y el Mayangna Yulbarangyang Balna (Elizabeth Salomón, Modesta Dolores, Tomasa Gómez, Demetrio Antolín y Gloria Fendly) -URACCAN, IPILC.

2 IELLab-Purdue University.

3 URACCAN, IPILC, Nicaragua. 
18

\section{Introducción}

Una de las particularidades que presenta el trabajo lingüístico en Nicaragua es que este fue solicitado desde dentro: fueron miembros de las propias comunidades los que en diferentes momentos del proceso de reclamo de sus derechos lingüísticos y culturales solicitaron la contribución de lingüistas. El segundo punto que caracteriza este trabajo lingüístico es que, como parte de un enfoque participativo que existía ya desde los tiempos de la Revolución, los propios miembros de la comunidad de habla se convirtieron en lingüistas. El tercer punto es que constituye un proceso de larga duración (en el momento de la escritura de este trabajo, cerca de 35 años) y sostenido (es decir, sin pausas ni lagunas durante ese periodo).

Así, el proceso de lo que en contextos académicos se denomina documentación adquirió en Nicaragua unas características bien especiales, en particular, porque se integra en un contexto estructural, que va creciendo y desarrollándose desde los años 80 , dedicado al fomento de las lenguas indígenas y autóctonas, lo que, en contextos académicos occidentales, se puede identificar como revitalización: el mantenimiento, fortalecimiento y desarrollo de las lenguas en su contexto cultural.

Dada esta coyuntura estructural excepcional (excepcional porque raramente se da), y las características particulares del trabajo lingüístico que se integra en esa estructura, este trabajo que aquí presentamos constituye un buen ejemplo de lo que podía ser un caso óptimo, una situación 'en el mejor de los casos' (un best-case scenario) para evaluar la relación entre el trabajo de documentación y su interacción con la revitalización lingüística. En particular, sería deseable identificar qué funciona y qué no funciona en este tipo de relación (entre documentación y revitalización) para poder hacer inversiones de futuro.

La estrategia que seguimos, pues, consiste en examinar una experiencia de documentación lingüística, de tipo tradicional, pero 
19

con un enfoque participativo comunitario que, incorporada en un proceso largo y sostenido, aumentaría las probabilidades de éxito, y que, al cabo de tres décadas, podría considerarse exitosa y positiva desde varios puntos de vista. Con ella en la mano, podemos evaluar los efectos que esa experiencia, positiva desde el punto de vista de la documentación, haya podido ejercer en el proceso de revitalización y fortalecimiento de la lengua.

Hablamos anteriormente de coyuntura y es claro, especialmente en el caso de Nicaragua, que hay toda una red de factores y actores que inciden en la salud de una lengua. Aquí no abordaremos todas las posibles variables (véase Benedicto et al., 2016 para un examen de una variedad de factores más amplia). Este trabajo que aquí presentamos es, más bien, una reflexión de lingüistas para lingüistas, el comienzo de un replanteamiento de cómo mejor interactuar con los otros protagonistas de la historia en los procesos de revitalización.

El presente escrito está organizado de la siguiente manera. En la sección 1 hemos establecido los parámetros que nos planteamos en el trabajo. En la sección 2 hacemos una presentación general del contexto lingüístico e histórico-social de Nicaragua. En la sección 3 presentamos las fases del proceso de documentación lingüística que se produjo en Nicaragua, bajo una perspectiva participativa, y su articulación con otras instituciones e iniciativas locales dirigidas al fomento de las lenguas. En la sección 4, ofrecemos una sistematización preliminar de los resultados observados. La sección 5, Haciendo balance, nos ofrece una reflexión crítica inicial de los resultados de ese proceso de documentación y finalmente en la sección 6, en Lecciones Aprendidas, hacemos balance de cómo los resultados de ese proceso de documentación han incidido o no en los procesos de revitalización y de las limitaciones de esa incidencia, de lo que funciona y lo que no funciona. 


\section{El contexto mayangna en Nicaragua}

\section{Ubicación geográfica}

En esta sección presentamos los mapas de la ubicación geográfica de las comunidades de habla mayangna, así como las lenguas vecinas con las que se relaciona.

Culturalmente, Nicaragua está dividida en dos zonas verticales: el oeste, identificado como el Pacífico, y el este, identificado como la Costa (véanse los rectángulos azul y rojo respectivamente en el mapa de abajo). El Pacífico es de mayoría cultural hispanohablante y alta influencia colonial española, e incluye algunos grupos indígenas que han podido mantener su identidad cultural pero no sus lenguas. La Costa es de mayoría cultural indígena, con una influencia colonial española muchísimo menor y una influencia colonial británica, y posteriormente estadounidense, muy prominente especialmente en la franja costera. En la Costa, actualmente, existen dos regiones autónomas: la Región Autónoma de la Costa Caribe Norte (RACCN, anteriormente conocida como RAAN, Región Autónoma del Atlántico Norte) y la Región Autónoma de la Costa Caribe Sur (RACCS, anteriormente conocida como RAAS, Región Autónoma del Atlántico Sur). En la RACCN, el miskitu es el grupo indígena dominante, y en la RACCS, el kriol es el grupo dominante, con el cual coexisten los grupos ulwa (familia misumalpa), rama (familia chibcha) y garífuna (lengua de contacto entre familia arawak y lenguas africanas), con presencia puntual miskita. ${ }^{4}$

4 Véase Benedicto, Shettle, y Mayangna Yulbarangyang Balna (2016), sección 2, y referencias ahí citadas, para una descripción de las interacciones y jerarquías sociolingüísticas entre los diferentes grupos y un resumen de la situación del marco legal en el país, concerniente a la situación de las lenguas. 
21

\section{Figura 1}

\section{Mapa linguíístico de Nicaragua}

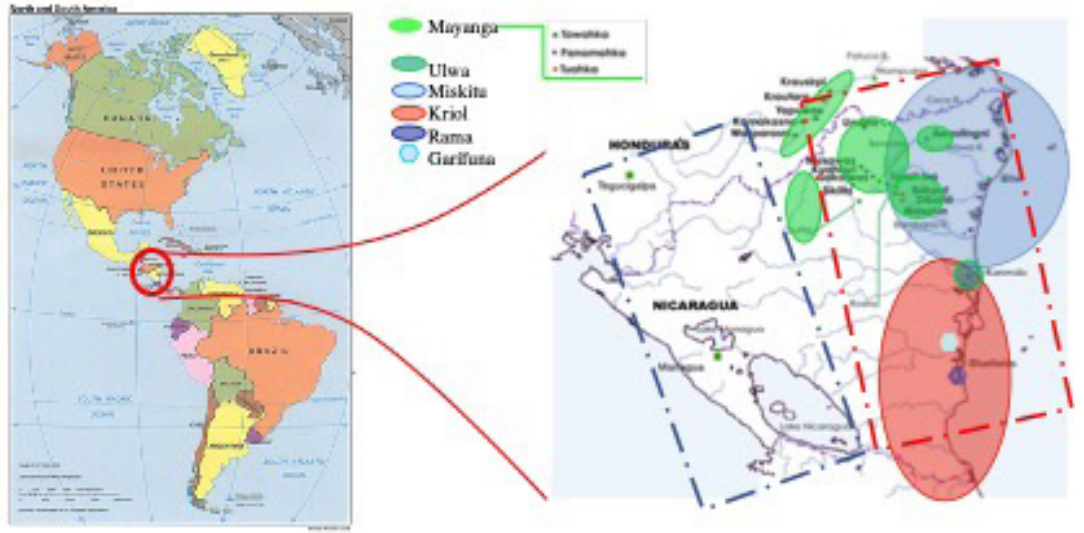

Adaptado de Benedicto y Hale, 2001 y de Benedicto et al., 2007.

Las comunidades mayangna se ubican en la Costa, en la RACCN, principalmente en su franja interior, en el centro del país (marcadas en verde en el mapa). Se pueden identificar varios grupos lingüísticos mayangna: el panamahka, mayoritario en Nicaragua, principalmente en la zona de Bonanza y las cuencas del río Waspuk y adyacentes; el tuahka, en la zona de Rosita, sobre la cuenca del río Bambana; el Yusku, sobre el cauce del río Bocay y adyacentes; el tawahka, en Honduras, sobre las cuencas del río Patuca.

\section{Breve contexto socio-histórico nicaragüense}

En 1979 se da en Nicaragua el triunfo de la Revolución que derroca al dictador Somoza. A raíz de esos eventos, que cambiaron radicalmente el país, se dieron una serie de iniciativas tendientes a mejorar, entre otras cosas, el nivel educativo de toda la población del país. Así se llevó a cabo la Campaña de Alfabetización, que consi- 
guió el reconocimiento de la UNESCO en 1980 (UNESCO, 2007; 25 Años). A raíz de estas iniciativas, se hizo evidente que la población indígena de la Costa no podía (ni debía) ser alfabetizada en español. Los reclamos de la población indígena, muy sensibilizada y concienciada de sus derechos y de la oportunidad histórica que se presentaba, hizo que se estableciera un cuerpo legal sustancial, entre otros y principalmente, un sistema de autonomía política para la Costa (Ley de Autonomía, Ley No. 28), un reconocimiento oficial de las lenguas (Ley de Lenguas, Ley No.162) y un sistema de educación en lengua indígena, el PEBI (Programa de Educación Bilingüe Intercultural), que debía cubrir desde el preescolar hasta la secundaria, pero que en la década de los 80 sólo llego a cubrir (parte de) la primaria y tendría que esperar hasta 2006 para ser incluido en el Sistema de Educación Nacional (Ley No. 582), como SEAR, Sistema de Educación Autonómico Regional (Sistema de Educación Autonómico Regional, 2001).

Tanto el aparato legal como el sistema educativo están basados en el concepto de derechos culturales y lingüisticos que llevó a la noción de relevancia cultural, la cual sería más tarde incorporada en el desarrollo de los estudios superiores en instituciones académicas como URACCAN.

\section{El proceso de Documentación Participativa del mayangna en Nicaragua}

Desde los comienzos de la década de los 80 , hubo presencia de lingüistas ${ }^{5}$ trabajando en la documentación de la lengua mayangna (al igual que de las otras lenguas en Nicaragua). Susan Norwood, en

5 Anteriormente, el único trabajo sistemático sobre las lenguas de la zona es el de Lehmann (1920). 
colaboración con activistas y docentes mayangna y en el marco de las actividades del CIDCA (Centro de Investigación y Documentación de la Costa Atlántica), escribió una Gramática Básica de la lengua en español (que, desafortunadamente, no llegaría a ser publicada hasta 1997; Norwood, 1988).

Al mismo tiempo, el profesor Luis Enrique López, que más tarde se incorporaría al PROEIB Andes en Bolivia, asesoraba los comienzos del PEBI. Y el grupo que se identificaría como Lingüistas por Nicaragua iniciaba, de la mano de Ken Hale, profesor en el MIT, investigaciones sobre la lengua y materiales para uso en las escuelas PEBI.

El PEBI Mayangna llegó hasta $4^{\circ}$ grado de primaria a finales de esta década, con libros de texto en todas las asignaturas escritos en lengua mayangna, incluyendo materiales de lectura y escritura. Muy importante para este proceso fue que vino acompañado de un trabajo de formación de maestros comunitarios que tenían en un principio una formación meramente empírica; las escuelas Normales en La Costa $^{6}$ iniciaron un proceso masivo de formación docente que, más tarde, continuaría la universidad URACCAN a nivel de educación superior. La educación PEBI, pues, disponía de materiales culturalmente relevantes, escritos en la lengua en aulas donde los maestros eran también miembros de la comunidad y hablantes de la lengua.

En la década de los 90, y a raíz de cambios en la escena política que tendrían repercusiones en el sistema escolar, el PEBI entra en modo de supervivencia. En esta época se cuenta, sobre todo, con el apoyo de ONG internacionales y se crea la universidad URACCAN ${ }^{7}$, con voca-

6 Véase Informe de Progreso Educativo, 2014 y 2015, así como McLean Herrera (2008).

7 URACCAN no es la única universidad en la Costa, pero sí la única que en esta década da cobertura de educación superior a la población Mayangna. 
ción de servicio a las poblaciones indígenas, y a la comunidad mayangna en particular, y portadora de la noción de educación relevante.

En URACCAN se crea el IPILC, el Instituto de Promoción e Investigación Lingüística y Revitalización Cultural, parte de la estructura general de la nueva Universidad en Institutos de Investigación con una visión comunitaria y participativa. Se desarrollan los programas de Licenciatura en EIB, en particular uno con enfoque mayangna. La oficina del IPILC en la zona mayangna organiza talleres de normalización de la lengua ${ }^{8}$ y coordina las ofertas de Licenciatura y Técnico Superior en EIB (Educación Intercultural Bilingüe), en formato por encuentros por estar enfocadas sobre todo a los maestros y maestras que trabajan en el sistema escolar PEBI, y mejor adaptarse así a sus condiciones laborales. A partir de las asignaturas de lengua mayangna que se ofertan como parte de la Licenciatura en EIB-mayangna, se comienza a formar, a finales de la década de los 90, un equipo de lingüistas autóctonos mayangna, el Mayangna Yulbarangyang Balna, que estará encargado en las dos décadas siguientes de un cuerpo substancial de trabajo lingüístico y de documentación de la lengua.

Con la infraestructura educativa de URACCAN y la colaboración de lingüistas externos (en particular, la última ola de Lingüistas

8 Por normalización se entiende el proceso de hacer el uso de la lengua 'normal', lo cual incluye un sistema ortográfico sistematizado. No incluye lo que en otros lugares se ha llamado un 'estándar' de lengua, que implica la elección de una variante sobre otra. En los procesos que se llevaron a cabo en Nicaragua se decidió respetar todas las variantes de la lengua y no primar una variante sobre otra (véase Benedicto, 2000; Benedicto et al., 2007; y Benedicto \& Mayangna Yulbarangyang Balna, 2018). Sí se decidió un sistema ortográfico que permitía escribir todas las variantes de la lengua usando las mismas convenciones (por ejemplo, la nasal velar sorda siempre se escribe $n g h$ independientemente de dónde se use en cada variante). 
por Nicaragua), se sientan las bases para un trabajo de documentación bajo un enfoque de Investigación-Acción Participativo (Benedicto et al., 2007), un componente sustancial del trabajo de URACCAN, y asumido como el enfoque por defecto por las comunidades participantes (que ya lo venían implementando desde la década anterior, a raíz de la concienciación surgida a partir de la Revolución). Este trabajo de documentación incluirá asimismo trabajo lingüístico descriptivo y teórico.

En la década de los 2000 y hasta mediados de la siguiente, y como resultado de las semillas plantadas a finales de la década anterior, se desarrollan una serie de actividades e iniciativas que constituyen el grueso del proceso de documentación y análisis lingüístico de la lengua mayangna hasta el momento.

Se crea e implementa en URACCAN, en la extensión existente en zona mayangna, una Especialización en Lingüística para lingüistas indígenas egresados de la Licenciatura EIB. A la par de ella, empieza a crecer el grupo de lingüistas mayangna (el Mayangna Yulbaranyang Balna), que funciona regularmente bajo un enfoque de Investigación-Acción Participativo (PAR) y que desarrolla su propia agenda de documentación y descripción de la lengua. Como implementación sustancial de ese enfoque Investigación-Acción participativo (PAR), se establece claramente que los lingüistas mayangna son actores en la creación de conocimiento sobre su lengua; que se trabaja para obtener un re-equilibro de poder (entre los colaboradores externos y los lingüistas mayangna); y que se han de establecer unos ciclos de transferencia del conocimiento que lleven de regreso a la comunidad los trabajos lingüísticos que se realizan y que, a su vez, la comunidad retroalimente el trabajo y la formación continua, tanto en las escuelas PEBI como de los lingüistas (incluyendo tanto lingüistas de la comunidad como colaboradores externos). Se enfatiza, por tanto, un círculo de interacción lingüistas-comunidad-formación continua. 
En esta época se lleva a cabo un Diccionario monolingüe mayangna, bidialectal (Panamahka y Tuahka), con definiciones en lengua mayangna, así como una Gramática de la lengua, escrita también en lengua mayangna ${ }^{9}$. Ambos trabajos se llevan a cabo con énfasis en el proceso, es decir, no se entienden como productos en aislamiento, sino como el resultado de un proceso creativo de formación del equipo de lingüistas autóctonos y sobre todo como (valga la redundancia) el proceso de creación de un proceso participativo que implique a las comunidades de manera relevante en la creación de conocimiento.

En paralelo, se van recopilando también materiales lingüísticos (i.e., textos en un sentido amplio) culturalmente relevantes, con participación directa y activa de comunitarios, que retroalimenten a las diferentes instancias de la comunidad (por ejemplo, las escuelas del PEBI; los procesos de recuperación de la memoria histórica, etc...), en lo que constituye un esfuerzo de encontrar una manera relevante de implementar el derecho al retorno a la comunidad de los materiales lingüísticos recopilados como parte de la documentación de la lengua ${ }^{10}$.

Con todo ello uno de los logros más sobresalientes de este periodo es la profesionalización del equipo de lingüistas mayangna, que acaban asumiendo muchas de las funciones que en décadas anteriores asumían los lingüistas colaboradores externos (por ejemplo, en la docencia en URACCAN).

Alrededor de mediados de la década de los 2010, comienzan a observarse cambios sutiles, pero con consecuencias potencialmente sustanciales, que podrían considerarse indicadores de deterioro.

9 Antolín, D., et al. (2011); y Dolores, M., et al. (en prensa).

10 Para unas reflexiones sobre la noción del derecho al retorno, véase Benedicto (2018). 
Se observan ciertos cambios en las escuelas comunitarias PEBI. La re-edición de los libros de texto en mayangna, que es responsabilidad del estado, deja de realizarse. Con ello las escuelas se encuentran en el dilema de o no usar libros de texto en las aulas, o usar los libros en español. Se escoge esta última opción y el efecto inmediato es que la lengua vehicular en la escuela cambia de mayangna a español. Lo más preocupante es que ello se da en comunidades lingüísticamente fuertes, lo cual lleva a preguntarse qué sucede en comunidades lingüísticamente vulnerables.

Por otro lado, los materiales de apoyo creados, tales como los Diccionarios y las Gramáticas, generalmente no acaban de llegar a las escuelas y las bibliotecas escolares, no se distribuyen y se acumulan en las estanterías del IPILC y URACCAN, con lo cual no llegan a ejercer ningún tipo de apoyo a la labor de los maestros. Los diccionarios se distribuyen, en ocasiones, como 'regalo corporativo' a representantes de instituciones, cumpliendo pues una función meramente simbólica y como marcador de estatus de lengua 'oficial'. En cuanto a las gramáticas, se organizan pequeños esfuerzos para socializar el contenido entre los maestros del PEBI y para facilitar su uso, pero son esfuerzos puntuales iniciados por el equipo de lingüistas mayangna y no-sistematizados ni asumidos por las instituciones locales (por ejemplo, el PEBI/MED, las escuelas Normales, o el IPILC/URACCAN).

Al mismo tiempo, se observa un desplazamiento profesional en el equipo de lingüistas mayangna, el motor de muchos de los cambios de la década anterior: algunos de ellos asumen puestos políticos o puestos de responsabilidad administrativa, con lo cual su disponibilidad para encargarse de tareas lingüísticas disminuye. Y, por otro lado, no se siguen los ciclos de formación lingüística (que fueron los que inicialmente generaron la creación del equipo de lingüistas mayangna) con estudiantes indígenas jóvenes que puedan dar continuidad. 
En URACCAN también se observan cambios, en las localizaciones geográficas de las licenciaturas y en transformaciones curriculares y desplazamientos en los programas universitarios (por ejemplo, el número de asignaturas relacionadas con la lengua disminuye en las licenciaturas EIB).

Es lo que se podría llamar una época de cansancio ${ }^{11}$, se dieron muchos esfuerzos anteriormente, a nivel personal y profesional, y esos esfuerzos comienzan a pasar factura. En general, se pierde empuje, y el poder del estado y las antiguas ideologias subyacentes ocupan espacio: ese momento de cansancio es aprovechado por las fuerzas atávicas, por las estructuras de poder antiguas, que nunca completamente desaparecieron, para ocupar posiciones de nuevo. En conclusión, se observa un abandono por pasiva de los avances obtenidos en las décadas anteriores. $\mathrm{Si}$ algo nos enseña este periodo es que no se puede bajar la guardia.

\section{Análisis de los resultados}

\section{Lo que se hizo}

En las páginas anteriores hemos podido observar que se llevaron a cabo una serie de acciones que podríamos considerar tradicionales en el campo de la documentación lingüística: se recopilaron textos, se realizó un diccionario y una gramática. Además, se estableció un estándar ortográfico (una revisión de modelos anteriores que

11 Hay otro factor que definitivamente tiene incidencia pero que, por lo que sabemos, no ha sido estudiado todavía, y es el incremento en los problemas territoriales, con una invasión sistematizada de los territorios indígenas Mayangna por parte de colonos hispano-hablantes procedentes del Pacífico. Este proceso, que es complejo, ha sido especialmente problemático para el caso de la lengua Tuahka que es la variedad más vulnerable. Para un análisis de la situación inicial, véase McLean Herrera (2008). En los siguientes 10 años, la situación se ha agravado sustancialmente. 
no reflejaban el sistema fonológico de la lengua) que respetaba las diferencias dialectales al tiempo que reflejaba la unidad de la lengua y que se aplicó masivamente al sistema educativo (a nivel de primaria, secundaria y universidad).

Todas estas actividades de documentación tradicionales se llevaron a cabo bajo un enfoque metodológico participativo, de investigación-acción. Este enfoque se utilizó de manera intencional, por diseño, no sólo para conseguir una mayor incidencia en el fortalecimiento de la lengua, sino, y quizá especialmente, por sus cualidades de justicia social, equidad y agentividad de los actores participantes.

\section{Lo que se observa después de más de 30 años, con respecto a la Revitalización}

Dadas esas condiciones de best-case scenario que hemos descrito más arriba, tocaba pues evaluar qué efectos pudiera haber conseguido ese proceso de documentación en la revitalización y fortalecimiento de la lengua.

La observación es, desafortunadamente, que existe un efecto cero en la revitalización de la lengua: las comunidades que perdían su lengua la han seguido perdiendo (por ejemplo, las comunidades Tuahka) y las comunidades que eran fuertes la mantuvieron, pero recientemente empiezan a observarse efectos de desplazamiento lingüístico (por ejemplo, en el territorio de Awastingni, y en el de Matumbak), bien hacia el miskitu o hacia el español (en zona urbana).

Los efectos positivos observados en ciclos anteriores (hasta mediados de los años 2000) se pierden a gran velocidad. Pero ¿cuáles han sido esos efectos? 
¿Qué efectos positivos se han podido observar?

Los primeros efectos positivos que se pudieron observar es la creación de recursos humanos profesionalizados. El equipo de lingüistas mayangna, el Mayangna Yulbaranyang Balna, fue altamente activo y productor durante 15 años y contribuyó a la profesionalización de muchas más generaciones de estudiantes indígenas en la universidad. Si tenemos en cuenta que la formación media de un maestro (empírico) en los años 80 era aproximadamente de $4^{\circ}$ grado, y hoy en día, después de varias generaciones de licenciatura en EIB-Mayangna, hay alrededor de 100 licenciados, sin contar los licenciados en otras disciplinas, y los graduados de másteres, el cambio es importante.

Se han dado, asimismo, efectos vitalizantes en las comunidades. Ello se observa en diversos factores: la aceptación en las comunidades de la educación bilingüe para la secundaria; la creación y una demanda en aumento de secundarias comunitarias; un cambio en la narrativa sobre el valor de la propia lengua y cultura en las comunidades; un incremento de la auto-conciencia lingüística y cultural en las comunidades; y un reconocimiento y re-valorización de los ancianos comunitarios como portadores del conocimiento (es decir, una concientización de que el conocimiento reside no en la cultura dominante, sino en la propia cultura, que la propia cultura es portadora de conocimiento valioso)

\section{Haciendo balance: una evaluación de los diferentes factores $y$ actores}

En esta sección hacemos un balance sistemático de los factores que hemos ido observando y describiendo en las páginas precedentes. Hemos escogido aquellos factores que se barajan más frecuen- 
31

temente en los trabajos sobre revitalización como factores de alta incidencia (positiva).

\section{La combinación de Documentación Tradicional + Investigación-Acción Participativa (PAR)}

Este combo manifiesta, definitivamente, efectos positivos, con ciertos efectos secundarios. Mayormente, los efectos positivos se reflejan en un beneficio a la profesión lingüística: se obtienen datos de alta calidad, y se procesan de manera más rápida y efectiva; se crean profesionales cuyas características responden a las necesidades del sistema académico occidental. Los efectos secundarios observados podrían caracterizarse como fuga de talentos: estos profesionales están altamente capacitados y reciben ofertas de trabajo que les apartan de la labor lingüística.

A pesar de los beneficios obtenidos, de este combo no se deriva, sin embargo, ningún efecto sobre la revitalización y el fortalecimiento de la lengua de manera natural. Estos efectos no se han podido observar: las tendencias (a la alta o a la baja) que existían con anterioridad siguen existiendo.

\section{El sistema escolar PEBI y su contribución}

¿Qué efectos se han podido observar como resultado de las escuelas PEBI? Aun siendo uno de los mejores sistemas educativos en Latinoamérica (es decir, uno en el que las deficiencias patentes en otros países no se detectan: los maestros y maestras provienen de las propias comunidades y, con contadas excepciones, siempre hablan la lengua de la comunidad; existen materiales en la propia lengua; existen programas de formación de maestros; etc...), el programa escolar revela sesgos que no son conducentes a proteger y reforzar la lengua. 
Entre ellos podemos mencionar que los modos de interacción entre maestro/as y estudiantes sigue siendo uno basado en patrones culturales occidentales; ello probablemente se deba a la falta de una formación de maestros sistemáticamente intercultural (la creación y mantenimiento de escuelas Normales con enfoque intercultural ha sido una tarea difícil $)^{12}$.

Y aunque ha habido esfuerzos para introducir una cosmovisión del mundo culturalmente relevante en los contenidos curriculares, la llamada contextualización del currículo escolar, la implementación concreta que se ha llevado a cabo queda, con contadas y honrosas excepciones, muy cercana a una traducción de los contenidos procedentes de la matriz del sistema español.

Asimismo, la propia estructura administrativa del PEBI responde a un modelo predominantemente occidental (el modelo nacional), al que se tiene que integrar de manera obligatoria.

En cuanto a su interacción con el trabajo lingüístico, uno de los problemas más preocupante es que no se ha conseguido que los trabajos lingüísticos (e.g., los diccionarios, la gramática, los textos, ...), que se iniciaron precisamente para alimentar al PEBI, se integren de manera efectiva y sistemática en los diferentes niveles de los programas curriculares de la lengua mayangna. A pesar de los esfuerzos de las personas implicadas, no se ha conseguido encontrar la mejor manera de conseguir esa integración.

El sistema escolar, por tanto, y a pesar de los esfuerzos de muchos de sus miembros, se mantiene como estandarte de la cultura dominante, en contenidos, estructuras y modos de enseñanza. Sin embargo, se podría argüir que, sin el sistema PEBI, la pérdida podía

12 Véase el Informe de Progreso Educativo, 2014 y 2015. 
haber sido mucho más rápida y contundente y que el gran logro del PEBI ha sido el frenar lo que hubiera sido una situación mucho peor.

\section{El papel de las instituciones}

Las instituciones han tenido un papel determinante, tanto a la hora de activar como a la hora de frenar. El papel que las instituciones de educación superior como URACCAN han tenido en este proceso ha sido crucial: han creado iniciativas, han canalizado recursos de diferentes orígenes, han catalizado energías y creatividad y han dado cauce, soporte y apoyo técnico a los diversos participantes en el proceso de fortalecimiento de las lenguas de la Costa nicaragüense. Muchas de las iniciativas que URACCAN creó en su momento han sido emuladas por otras instituciones. También es cierto, sin embargo, que las instituciones cambian, cambian las personas que las dirigen y cambian las prioridades, y lo que en un momento pudo ser un buen modelo con resultados excelentes, en una situación futura puede no encontrar sustento. Ello requiere una evaluación y adaptación continua, para poder obtener los mejores resultados posibles.

Las instituciones de educación básica, como el PEBI y el Ministerio de Educación a nivel nacional, son también cruciales para que la ecuación final produzca los efectos deseados. También aquí los cambios en el contexto político-social, en las prioridades de dirigentes y de técnicos, y en la asignación de recursos (por ejemplo, para sustentar la reedición de libros de texto de manera sostenible) son elementos que necesitan ser monitoreados, evaluados y ante los cuales hay que producir adaptaciones.

Finalmente, es importante remarcar el papel que ejercen las instituciones políticas, las tensiones entre ellas (a nivel nacional y regional), el financiamiento del que se dispone (o no) para financiar 
iniciativas de apoyo a la lengua, y el soporte institucional que pueden dar a las estructuras educativas.

Si tales instituciones existen en un contexto propicio, serán elementos clave en el proceso de fortalecimiento lingüístico. Si no existen, o el contexto no es propicio, las iniciativas deben adaptarse y diseñarse en consecuencia.

\section{Lecciones aprendidas}

Quizá una de las principales lecciones aprendidas, y uno de los primeros mensajes que este trabajo quisiera compartir, es que es necesario y absolutamente crucial, en este campo de la revitalización y el fortalecimiento de las lenguas, hablar de aquello de lo que no hablamos: lo que no funciona.

Hablar de los éxitos, de lo que ha funcionado en este o aquel contexto es ciertamente útil. Pero hablar de lo que no funcionó e intentar averiguar por qué no funcionó es igualmente útil, si no más. Puede impedir tropezar dos veces (¡o más!) con la misma piedra, puede ahorrarnos recursos preciosos o, sencillamente, puede hacernos conscientes de las posibles piedras en el camino para estar listos y preparados. No quiere decir que no hayamos de tomar ciertos riesgos, experimentar, probar esto y aquello. Podemos experimentar con diferentes tipos de paracaídas, por así decir, pero sabemos que saltar sin paracaídas no funciona. Identificar lo que puede constituir 'saltar sin paracaídas' o 'usar un paracaídas no adecuado a la altura, los vientos, etc..' es aquello por lo que abogamos aquí.

Así que, en esta última sección, exponemos a grandes rasgos lo que hemos aprendido que no funciona. 
1. Para empezar, el primer mito que quisiéramos reportar es el de que la documentación tradicional por sí misma y en sí misma pueda revitalizar. No lo hace. En lo que hemos podido observar en este periodo largo y sostenido de trabajo, y lo que hemos podido corroborar en otras experiencias, es que la documentación tradicional tiene un efecto neto 'cero' en revitalización y fortalecimiento. En el caso del mayangna en Nicaragua, las variedades lingüísticas que gozaban de vitalidad la continúan teniendo, las que no la tenían, la siguen perdiendo.

Ello no quiere decir que los materiales y el conocimiento que se obtiene a través de la documentación no sirva para generar y diseñar acciones de revitalización y fortalecimiento. Lo que quiere decir es que esas acciones se tienen que diseñar explícitamente para la revitalización y el fortalecimiento.

La segunda parte de lo que hemos podido descubrir es que esas acciones explícitas de revitalización y fortalecimiento han de salir de dentro de la cultura asociada con la lengua. Preparar materiales escolares al estilo occidental no suele aportar mucho. Los materiales acaban acumulando polvo y siendo olvidados. Se han de conectar esos materiales (que han de ser necesariamente culturalmente relevantes) a acciones, iniciativas y prácticas culturales que estén enraizadas en la vida comunitaria. Si existe un sistema escolar que pueda recibir esos materiales, se ha de proporcionar una formación continua y sostenida a los maestros y maestras que les permita integrar esos materiales en sus prácticas diarias (que han de estar sintonizadas con la cultura) ${ }^{13}$.

13 Buenos ejemplos de este tipo explícito de acciones para la revitalización, en un contexto culturalmente relevante, salieron en diferentes contribuciones de jóvenes activistas durante el I Congreso Internacional Contacto, Documentación y Revitalización de Lenguas en Desplazamiento en Hispanoamérica: Desafios en la Diversidad II en San José de Costa Rica (febrero, 2018). Un ejemplo relevante 
Por tanto, la común aserción que 'esta documentación de la lengua $\mathrm{X}$ contribuye a su revitalización' es inválida, a menos que incluya acciones directamente y específicamente diseñadas, de manera culturalmente relevante, para la revitalización.

2. Un segundo punto a considerar es la contribución de las metodologías participativas y de interacción y compromiso con la comunidad de hablantes. Estas sí que han podido marcar un efecto positivo, pero esos efectos deben matizarse.

Ciertamente, la participación activa y directa de un grupo de lingüistas comunitarios ha tenido un efecto positivo y vitalizante en los procesos de conversación interna sobre el valor de la lengua. Ello se ha visto en las actitudes de la propia comunidad hacia su propia lengua, en la auto-conciencia sobre el valor de la lengua y cultura, y en los cambios que se han observado en las 'narrativas' iniciales sobre ellas.

Estos efectos, sin embargo, no han podido evitar los efectos regresivos que se han observado más recientemente, por ejemplo, en las escuelas PEBI (y que sean posiblemente debidos a factores de ideologías subyacentes profundas).

3. El tercer punto está relacionado con los ciclos vitales. La conexión entre los ciclos vitales de documentación y revitalización es clave para un éxito continuado. Un cierto tipo de fórmula puede funcionar bien en referencia a un contexto determinado, pero cambios en ese contexto pueden conducir a un rediseño de las iniciativas. En tales casos se ha de conectar los dos sistemas para que no se produzca ruptura.

En el caso de Nicaragua, los procesos de formación que funcionaron muy bien durante una década desde finales de los 90, no

de integración coherente en el sistema escolar lo encontramos, por ejemplo, en el trabajo de Ruiz Chaverri (2018a y 2018b). 
tuvieron continuidad cuando el contexto cambió. Y, en consecuencia, no se produjo el relevo generacional que hubiera proporcionado continuidad a las iniciativas de fortalecimiento de la lengua. En palabras de Melba McLean, líder mayangna y directora del Centro de Investigaciones y Desarrollo de la Costa Atlántica (CIDCA), hay que asegurar que existe un semillero que proporcione las plantas del mañana.

\section{Referencias bibliográficas}

25 años de la Cruzada de Alfabetización: "El país entero fue una gran escuela". 2005. Envío, 289. http://www.envio.org.ni/articulo/3004 (acceso: 24 de junio de 2014)http://www.envio.org.ni/articulo/3026 (Versión en inglés, acceso: 24 de junio de 2014).

Antolín, D., Salomón, E., Gómez, T., Taylor, C., Genaro, J., Miguel, B., Feliciano, M.C., Frank, E., McLean, M., \& Benedicto, E. (2011). Mayangna Yulpuyang [Diccionario Mayangna] Volumen 1, Panamahka. Rosita, Nicaragua: Alcaldía de Rosita y Junta de Andalucía-Programa de Reconstrucción post-Félix Nicaragua Fase I.

Benedicto, E. (2000). A community's solution to some literacy problems: The Mayangna of Nicaragua. En Ostler, N. y Rudes, B. (Eds.), Proceedings of the IV Conference on 'Endangered Languages and Literacy' (pp. 19-24). Charlotte, NC: Foundation for Endangered Languages.

(2018). When Participatory Action Research (PAR) and (Western) Academic Institutional Policies do not align. En Bischoff, S. y Jany, C. (Eds.), Perspectives on Language and Linguistics: Community-Based Research (pp. 24-41). Berlín: De Gruyter Mouton.

Benedicto, E., \& Mayangna Yulbarangyang Balna (2018). La tercera vía de 'normalización': sistematización de escritura vs lengua estándar. Presentada en el 56 Congreso Internacional de Americanistas, ICA, Simposio Norma y estandarización en lenguas indígenas de América. Salamanca, 15-20 de julio de 2018.

Benedicto, E. \& Hale, K. (2001). Mayangna: A Sumu Language and Its Variants. En Benedicto, E. (Ed.), The UMass Indigenous Languages Volume (pp. 75-106). UMOP [University of Massachusetts Occasional Papers], 20.

Benedicto, E., Shettle, A., \& Mayangna Yulbarangyang Balna (2016). Linguistic ideologies as a Crucial Factor in Language Revitalization: A Look at the 
Mayangna Languages of Nicaragua. En Pérez, G., Rogers, Ch. y Rosés Labrada, J.E. (Eds.), Latin American Contexts for Language Documentation and Revitalization (pp. 305-344). Berlín: De Gruyter Mouton.

Benedicto, E., Antolín, D., Dolores, M., Feliciano, M., Fendly, G., Gómez, T., Miguel, B., \& Salomón, E. (2007). A model of Participatory Action Research: the Mayangna linguists' team of Nicaragua. En Maya Khemlani, D., Ostler, N. y Dealwis, C. (Eds.), Proceedings of the XI FEL Conference on 'Working Together for Endangered Languages - Research Challenges and Social Impacts' (pp. 29-35). Kuala Lumpur, Malaysia: SKET, University of Malaya y Foundation for Endangered Languages.

Dolores, M., Fendly, G., Gómez, T., Poveda, C., McLean, M., \& Benedicto, E. (en prensa). Mayangna Yulpuyang [Diccionario Mayangna] Volumen 2, Tuahka. Rosita, Nicaragua: Alcaldía de Rosita y Junta de AndalucíaPrograma de Reconstrucción post-Félix Nicaragua Fase I.

Informe de Progreso Educativo. Calidad y Equidad para el Desarrollo Humano. Nicaragua. (2014). OPREAL, EDUQUEMOS, IBIS, Telefónica.

Informe de Progreso Educativo. Educación Intercultural Bilingüe con Calidad y Equidad para la Autonomía. Región Autónoma de la Costa Caribe Sur. Nicaragua. (2015). URACCAN, EDUQUEMOS, IBIS, Telefónica.

Lehmann, W. (1920). Zentral-Amerika, Teil I, Die Sprachen Zentral-Amerikas in ihren Beziehungen zueinander sowie zu Sud-Amerika und Mexiko. Berlin: Verlag Dietrich Reimer.

Ley No. 162. 1996. Ley de uso oficial de las lenguas de las comunidades de la Costa Atlántica de Nicaragua. (1993). La Gaceta, 132.

Ley No. 28. 1987. Estatuto de autonomía de las Regiones de la Costa Atlántica de Nicaragua. (1987). La Gaceta, 238.

Mayangna Yulbarangyang Balna \& Benedicto, E. (2011). Mayangna Yulni Barangwa Lâni [Gramática Mayangna]. Rosita, Nicaragua: Alcaldía de Rosita y Junta de Andalucía-Programa de Reconstrucción post-Félix Nicaragua Fase I.

McLean Herrera, G. (2008). La educación intercultural bilingüe: el caso nicaragüense. Colección Libros FLAPE 26. Buenos Aires: Fund. Laboratorio de Políticas Públicas.

Norwood, S. (1988/1997). Gramática de la lengua sumu. Managua: Centro de investigaciones y documentación de la Costa Atlántica, CIDCA.

Ruiz Chaverri, D. (2018a). La apropiación de la ortografía en comunidades indígenas: del dicho al hecho hay mucho... vacío metodológico. Ponencia presentada en el I Congreso Internacional Contacto, Documentación y Re- 
vitalización de Lenguas en Desplazamiento en Hispanoamérica: Desafios en la Diversidad II. Febrero, 2018. San José, Costa Rica.

(2018b). La apropiación de la ortografía: un encuentro entre la reflexión metalingüística y las prácticas socioculturales. Ponencia presentada en el $56^{\circ}$ Congreso Internacional de Americanistas. Salamanca, España. Julio 2018. Simposio: Norma y estandarización en las lenguas indígenas de América. Sistema Educativo Autonómico Regional (SEAR) (2001). URACCAN, Consejos Regionales Autónomos RAAN/RAAS, Gobiernos Regionales Autónomos RAAN/RAAS.

UNESCO (2007). Memoria del mundo: La cruzada nacional de alfabetización. Nicaragua. http://www.unesco.org/new/es/communication-and-information/flagship-project-activities/memory-of-the-world/register/fulllist-of-registered-heritage/registered-heritage-page-6/national-literacycrusade/(24 de junio 2014). 